\title{
Por medio de un sistema informático en las escuelas de conducción.
}

By means of a computer system in driving schools.

Velasteguí López Efraín. ${ }^{1}$, Bravo Córdova Brayan Daniel. ${ }^{2}$

DOI: https://doi.org/10.33262/visionariodigital.v1i4.256

\section{Resumen.}

En el presente ensayo se va a tratar un tema importante para las autoescuelas o escuelas de conducción como para la informática y otras especialidades ya que implica un ámbito muy importante en lo tránsito-vial, en sistemas y la recopilación de información que será útil en el caso de certificar a un nuevo conductor o chofer. Se busca que en todas las autoescuelas se certifiquen a alumnos que aseguren una mejor circulación vehicular, brinden un buen servicio (en el caso de los choferes), seguro y eficiente, y reducir considerablemente los accidentes de tránsito, que en estos tiempos ha aumentado desmesuradamente. La gestión mediante un sistema informático en las autoescuelas, es lo que conlleva a llevar un buen registro y control de los alumnos, ya que, existe un número que son certificados, sin conocimiento alguno, ya sea por actos injustificables como son: sobornos, trampas, hurto, entre otros., llegando incluso a ocupar cargos que pueden involucrar a otras vidas. En este tema se va a mencionar asuntos esenciales que serán vitales para proyectar la idea que se ha planteado para reducir estos trágicos casos, que ya va llevando a más personas en este año. Cada tema es investigado y analizado de diferentes autores, páginas web, revistas, diarios o libros citados, con el fin de que este ensayo sea justificado, los mismos que llevan información necesaria para la ingeniería de sistemas, que también implicara en lo profesional. Para finalizar se dejará las debidas conclusiones que parezcan más oportunas para la práctica del tema.

Palabras clave: Sistema, informática, gestión, autoescuela, control, educación, programa, aplicación.

\footnotetext{
${ }^{1}$ Universidad Técnica de Cotopaxi Ext La Maná, Cotopaxi, Ecuador, le.velastegui@uta.edu.ec ${ }^{2}$ Universidad Técnica de Cotopaxi Ext La Maná, Cotopaxi, Ecuador, brayanb09@ gmail.com 


\begin{abstract}
.
In the present essay an important topic will be treated for driving schools or driving schools as well as computer science and other specialties since it involves a very important area in the transit-road, in systems and the collection of information that will be useful in the Case of certifying a new driver or driver. It is intended that all driving schools certify students to ensure a better vehicular circulation, provide good service (in the case of drivers), safe and efficient, and significantly reduce traffic accidents, which in these times has increased disproportionately. The management through a computer system in the driving schools, is what leads to a good record and control of students, because there is a number that are certified, without any knowledge, either by unjustifiable acts such as: bribes, cheating , robbery, among others., even occupying positions that may involve other lives. In this issue we will mention essential issues that will be vital to project the idea that has been proposed to reduce these tragic cases, which is already leading to more people this year. Each topic is researched and analyzed by different authors, web pages, magazines, journals or books cited, in order that this essay is justified, the same ones that carry necessary information for systems engineering, which also imply in the professional. To finalize it will leave the due conclusions that seem more opportune for the practice of the subject.
\end{abstract}

Keywords: System, informatics, management, driving school, control, education, program, application.

\title{
I. Introducción.
}

\section{Sistema.}

Según Castillo L. (2005)., Kuri J. (2018)., Pérez G. y Rodríguez P. (2016)., Anónimo (2018) Un sistema es un tema con diversas definiciones, por un lado, se menciona que está desarrollada de manera intuitiva debido al quehacer diario de la vida personal, aunque su concepto principal es que es un conjunto de elementos que interactúan con un objetivo común entre ellos, donde estos elementos por una parte son físicos y por otra son métodos, reglas y procedimientos que determinan la forma de intervenir de los elementos físicos. Se menciona que un sistema está integrado por diferentes factores agrupados de tal manera que constituya un lógico y funcional, que es mayor que la suma de esas unidades. Por ultimo también se menciona que un sistema es un conjunto de funciones, virtualmente referenciada sobre ejes, ya sean estos reales o abstractos. 


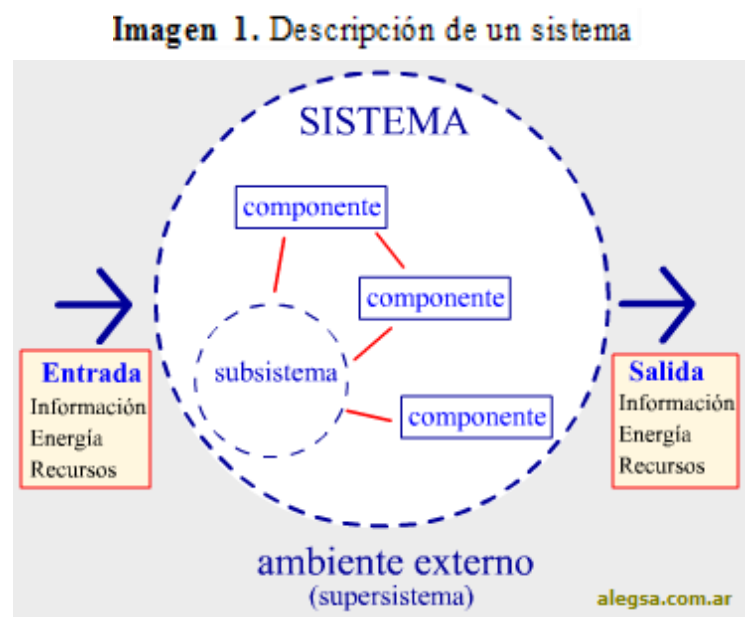

Autor: Leandro Alegsa

Fuente consultada: http://www.alegsa.com.ar/Dic/sistema.php

En lo mencionado por estos autores, un sistema es un esquema organizada de diferentes elementos, siendo de tipo físico y un proceso, método y regla que intervengan la formación de este tipo, para completar un objetivo propuesto el cual es definido por decisión del usuario, estos pueden influir en la tecnología, en la administración, en la economía, entre otros. Hay que tomar en cuenta que la definición de sistema está basada a todo lo relacionado a la realización de actividades y necesidades diarias de la vida de las personas, desde pequeños problemas, hasta problemas magnos, los cuales se presentan en todo ámbito de cada persona. A demás de que un sistema también se lo define como una amalgama de funciones, ya sean estos reales o abstractos, aunque esta parte abarca en si a todo el significado global de sistema.

\section{Informático o Informática.}

Según Villazan F. (2010)., Junta de Andalucía (2011)., HellHacker (2018)., Anónimo (2018) La informática se ha relacionado con el término de computación. Su nombre se deriva de dos términos: información (conjunto de datos organizados) y automática, en base a ello se ha mencionado que es un conjunto de conocimientos científicos y técnicas que hacen posible el tratamiento y manipulación automática de la información por medio de computadoras. Se menciona que se refiere al procesamiento automático de información mediante dispositivos electrónicos y sistemas computacionales, reuniendo muchas técnicas que el hombre desarrollo, aspirando a potenciar sus capacidades de pensamiento, memoria y comunicación. También que combina aspectos teóricos y prácticos de la ingeniería electrónica, matemática lógica, y comportamiento humano. Estos aspectos de la informática cubren desde la programación y la arquitectura informática hasta la inteligencia artificial y la robótica. 


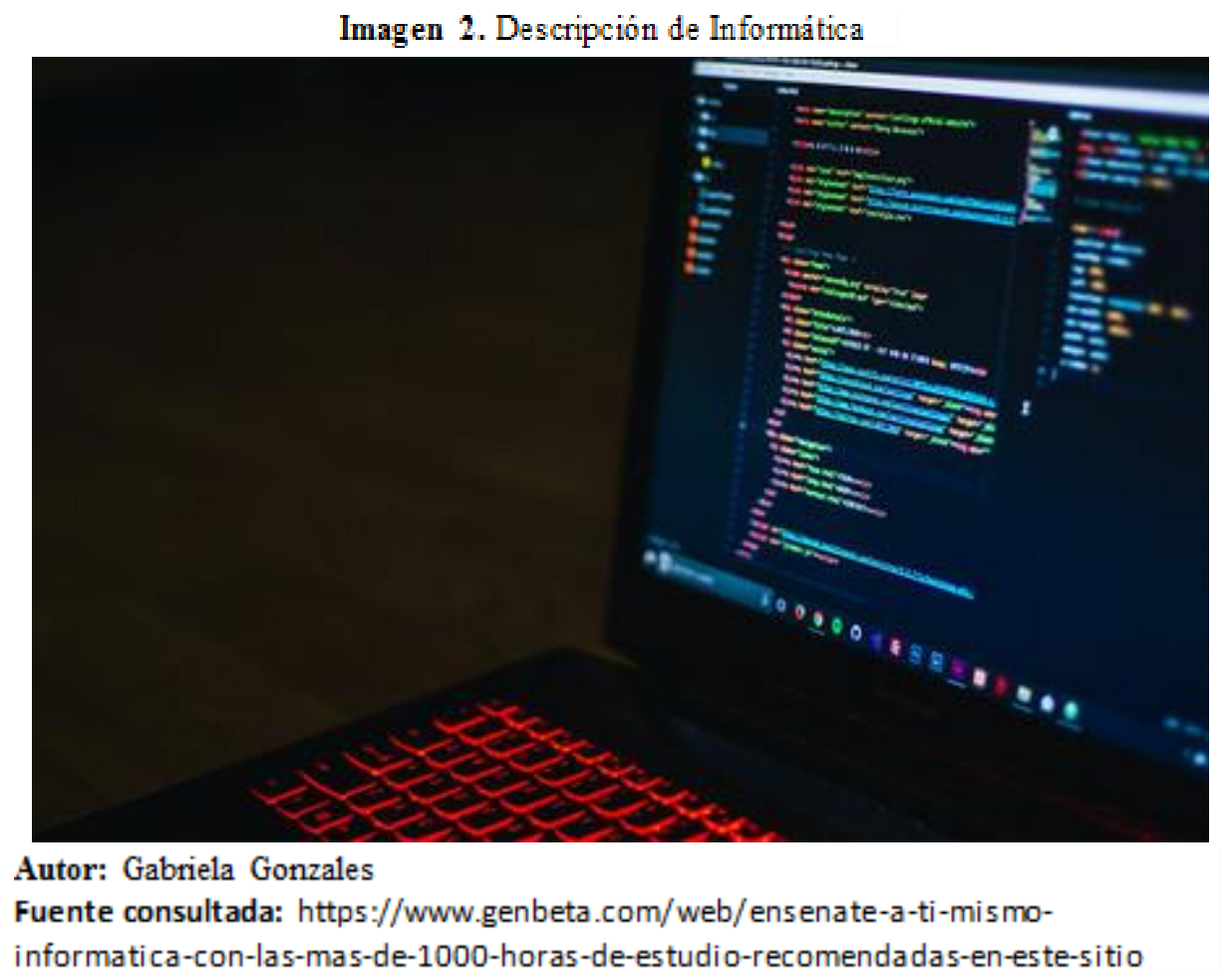

En lo mencionado por estos autores se dice que la informática está presente en las actividades de las personas, por la manipulación de información y tratarla de acuerdo al usuario que la posea, todo esto por medio de una computadora, además de estar relacionado a la lógica matemática, a la electrónica que son vitales para un diseño estructurado relacionado al comportamiento del ser humano (robots). La informática también se dice que puede ser utilizada en diferentes ámbitos profesionales, ya sea en la salud, la comunicación, transporte, en la gestión de los negocios, para almacenar grandes o pequeñas cantidades de información, y en el control de procesos. Comenzando por la programación y el diseño de un esquema, incluso llegando a usar grandes recursos como la inteligencia artificial (IA) y la robótica.

\section{Sistema informático.}

Ya comprendido el significado de sistema e informática, se dará a conocer que es un sistema informático.

Según Villazán F. (2010)., Junta de Andalucía (2011)., Chacón J. (2018)., Ibáñez D. (2018)., Anónimo (2018) Un sistema informático es un conjunto de elementos físicos o también conocidos como hardware que son necesarios para la explotación de las aplicaciones informáticas o software, o se dice que un sistema informático que hacen posible el tratamiento automático de la información, los cuales conforman componentes físicos, lógicos y humanos. También se dice que es un conjunto de elementos para la realización y utilización de aplicaciones informáticas, integrado por elementos principales: equipos, programas, firmware y personal informático. Además, se menciona que la información con el sistema informático es capaz de almacenar y difundir los conocimientos. Un sistema 
informático como utilizador fina se desempeña en dos actividades que son fundamentales: toma de decisiones y control.

Imagen 3. Elementos de un Sistema Informático
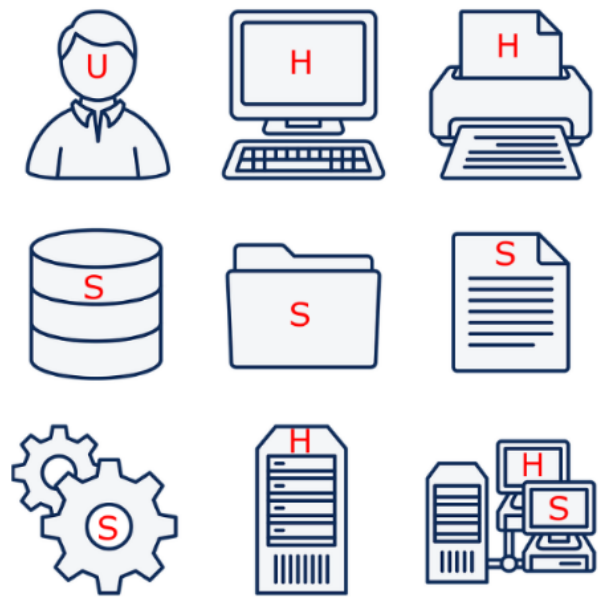

Autor: Guillermo Aran

Fuente de imagen: http://tecnologialaran.blogspot.com/2017/06/sistemas-procesos-y-

Lo antes mencionado por los autores nos dice que un sistema informático es una utilización de aplicaciones y programas mediante un conjunto de elementos físicos para el tratamiento automatizado de la información. Un sistema informático permite al usuario interactuar con la máquina para procesar, almacenar y difundir toda la información. Además, los programas o software no pueden ser utilizado sin un equipo físico, periférico o hardware, así mismo estos equipos sin un usuario son inservibles y sin una orden no son capaces de realizar ciertas acciones, es decir que todos los componentes van en cadena, uno apoyándose del otro. Como punto final se hace tomar en cuenta la toma de decisiones y control, porque los sistemas van recibiendo actualizaciones cada día y una buena decisión puede ir adaptando esas actualizaciones y con el control adecuado mantenerlo estable.

\section{Enseñanza de las escuelas de conducción.}

La autoescuela o escuela de conducción es una institución que enseña, prepara y certifica a una persona mayor de edad a circular por las vías usando un vehículo motorizado. Para identificar si está o no certificado, a la persona aprobada se le otorga un documento (licencia), con el cual puede identificarse hacia un agente de tránsito.

Según Flores Corzo V. (2018) Las clases prácticas que imparten las autoescuelas tienen como misión preparar a sus alumnos sobre las situaciones cotidianas de la circulación. Unos aspirantes que tendrán que demostrar al volante el día del examen, si están preparados como conductores a desenvolverse en una vía publica junto con el resto de usuarios, ciclistas y peatones que comparten el mismo espacio. 
Imagen 4. Aula de una autoescuela o escuela de conducción

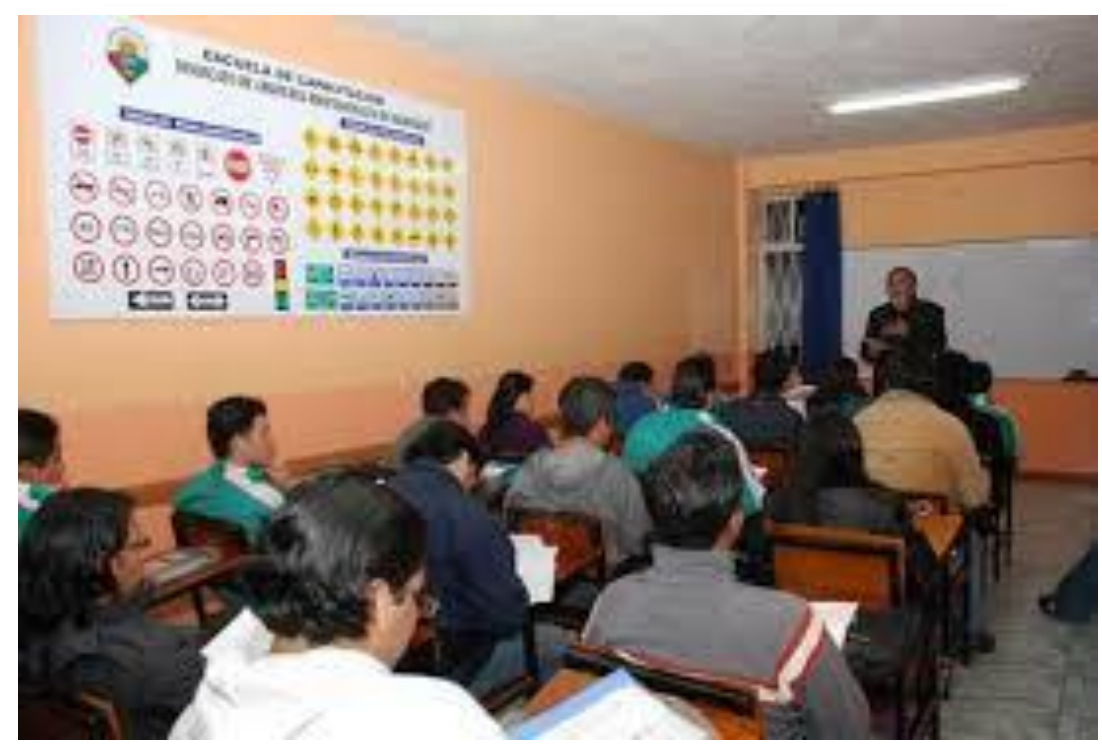

Autor: Diario LaHora

Fuente de imagen: https://lahora.com.ec/noticia/1101188281/universidades-serc3a1n-escuelasde-conduccic $3 b 3 n$

La mayor parte de los estudiantes que desean entrar a obtener una licencia van con la idea de que aprenderán a derrapar y a ir a toda velocidad en el vehículo, aunque lo más esencial es la seguridad y eso es lo que toman en cuenta los profesores en sus enseñanzas para que exista un lazo de paz entre el conductor, el peatón y el ciclista.

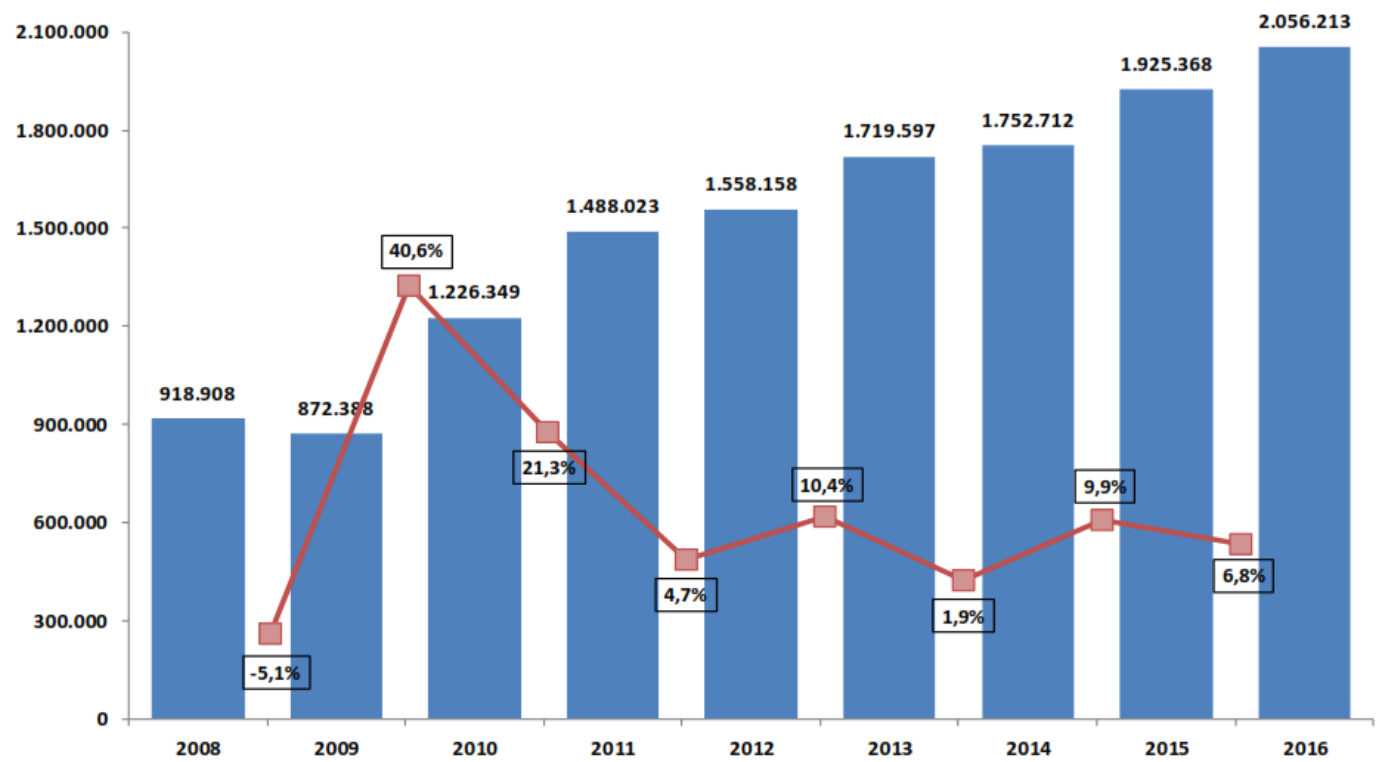

Figura 1. Número de vehículos matriculadosentre los años 2008 y 2016

Autor: Agencia Nacional de Tránsito

Fuente de imagen: http://www.ecuadorencifras.gob.ec/documentos/web-

inec/Estadisticas_Economicas/Estadistica\%20de\%20Transporte/2016/2016_AnuarioTransportes_\%20Principal es\%20Resultados.pdf 


\section{Problemas con los conductores.}

Aparte de que ya se realizan nuevas leyes de tránsito, control y recalificación en conductores y profesionales, además de una revisión vehicular, todo para poder reducir el número de accidentes realizados. El número no ha bajado, ya que no acatan las disposiciones puestas anteriormente. Blog de la Autoescuela (2013) afirma:

Los choferes de Ecuador son quienes provocan los accidentes de tránsito, debido a que les gusta conducir a límites de velocidad superiores a los permitidos, la ley establece que nadie puede circular a más de 50 kilómetros por hora en las calles de una ciudad ecuatoriana.

Tabla 1. Accidentes de Tránsito del país en el afio 2018

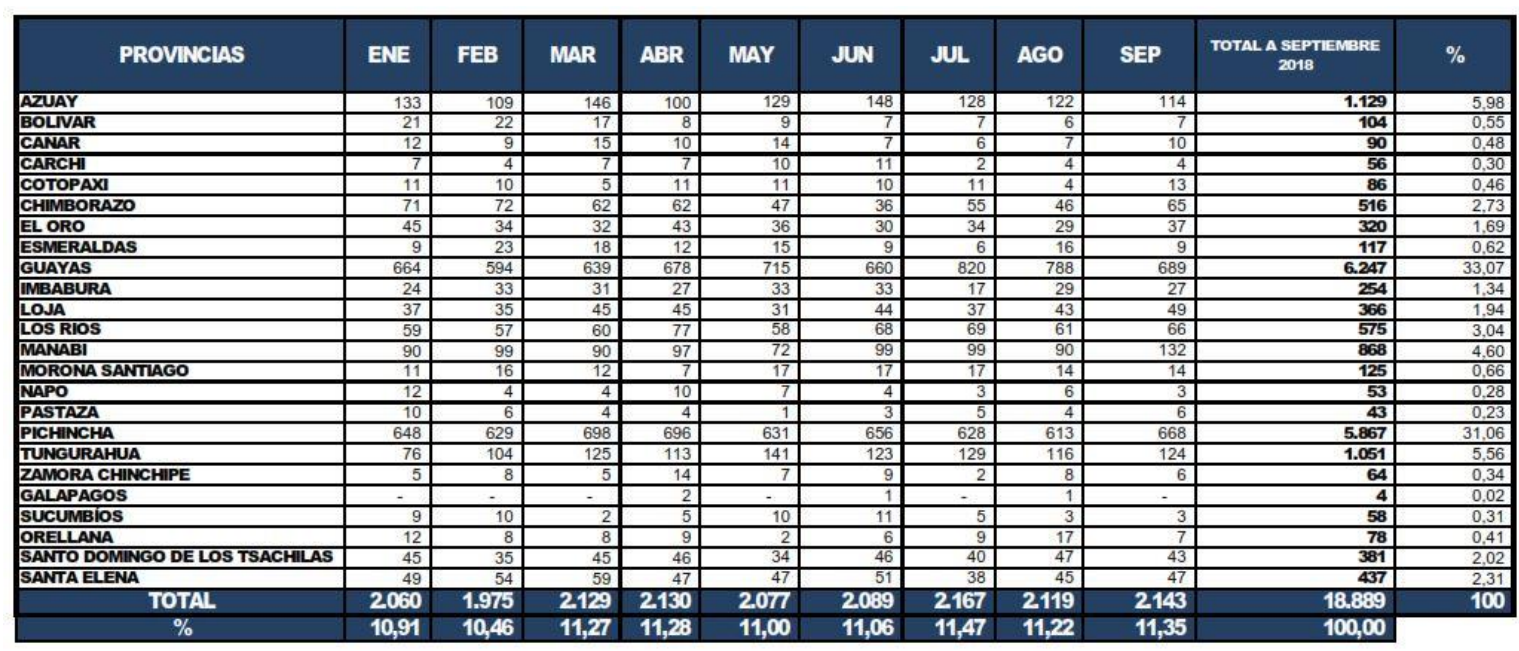

Autor: Agencia Nacional de Tránsito

Fuente de imagen: https://www.antgob.ec/index.php/descargable/file/5872-siniestros-septiembre-

Este dato afirma que choferes, como por ejemplo de buses ya sean interprovinciales o rurales, camiones, tráileres, entre otros., hacen caso omiso a la ley dispuesta, no darle una revisión adecuada al vehículo o no realizar un control de sus conocimientos, se notifican diferentes accidentes de esta índole. En ciertas autoescuelas se ha visto que existen sobornos para ciertas pruebas, trampas, entre otros factores que afectan y aprueban a conductores no calificados entrando al mundo vial automovilístico y llevando a casos peores.

\section{Importancia del sistema informático en una autoescuela.}

Ya mostrado todos estos temas, se da la idea de que un sistema informático que permita llevar un control y mantenimiento de los alumnos de la autoescuela, revisando un registro del mismo, ya sea asistencia, realización de las pruebas, teóricas, prácticas y psicológicas, todo mediante ese sistema que puede demostrar más veracidad, y reduciría considerablemente el número de accidentes que suceden al día.

En Ecuador se menciona que existen más de 100 autoescuelas, cada una ofreciendo un buen servicio y educación a sus alumnos. Aunque hasta ahora no se ha encontrado alguna que lleve la gestión por medio de un sistema informático, haciendo que algunas no tengan 
veracidad ni transparencia, ya que pueden existir cambios de dudosa procedencia por medio de un tercero o del mismo alumno, ya sea con el objetivo de pasar y obtener su licencia.

Autoescuelas del extranjero FOCOSUR, E-SCUELA, Lineal S.L., entre otros, ya han dado un paso a este sistema informático y muestran las mejoras de las mismas, tanto en sus alumnos como en sus pruebas. "Controlar los riesgos que puedan existir y las oportunidades de mejora que se nos pudiesen presentar e ir mejorando continuamente en nuestro funcionamiento" (Moriano Expósito, 2016). Es decir, que este sistema además de mostrar calidad frente a todos los usuarios que van a usar, cumplirá con el régimen legal de tránsito, garantizará alumnos de calidad para cumplir con el régimen ya mencionado.

\section{EI Sistema Informático en autoescuelas del cantón La Maná.}

En el cantón La Maná, de la provincia de Cotopaxi, está el Sindicato de Choferes Profesionales del Cantón La Maná, el cual está centrado en capacitar a alumnos deseosos de aprender y ocupar algún cargo en el ámbito profesional, camionetas, taxis, entre otros. También están los diferentes sindicatos que van dirigidos a las Moto-taxis, que este medio es el más utilizado en todo el cantón y lleva consigo diversas Cooperativas. Además, la Escuela de Capacitación de Conductores no Profesionales "Conrespop La Maná", como su nombre lo indica está centrado en capacitar a alumnos deseosos de aprender sin ocupar los cargos profesionales ya mencionados.

Imagen 6. Sindicato de Choferes Profesionales del Cantón La Maná en Pruebas Teóricas

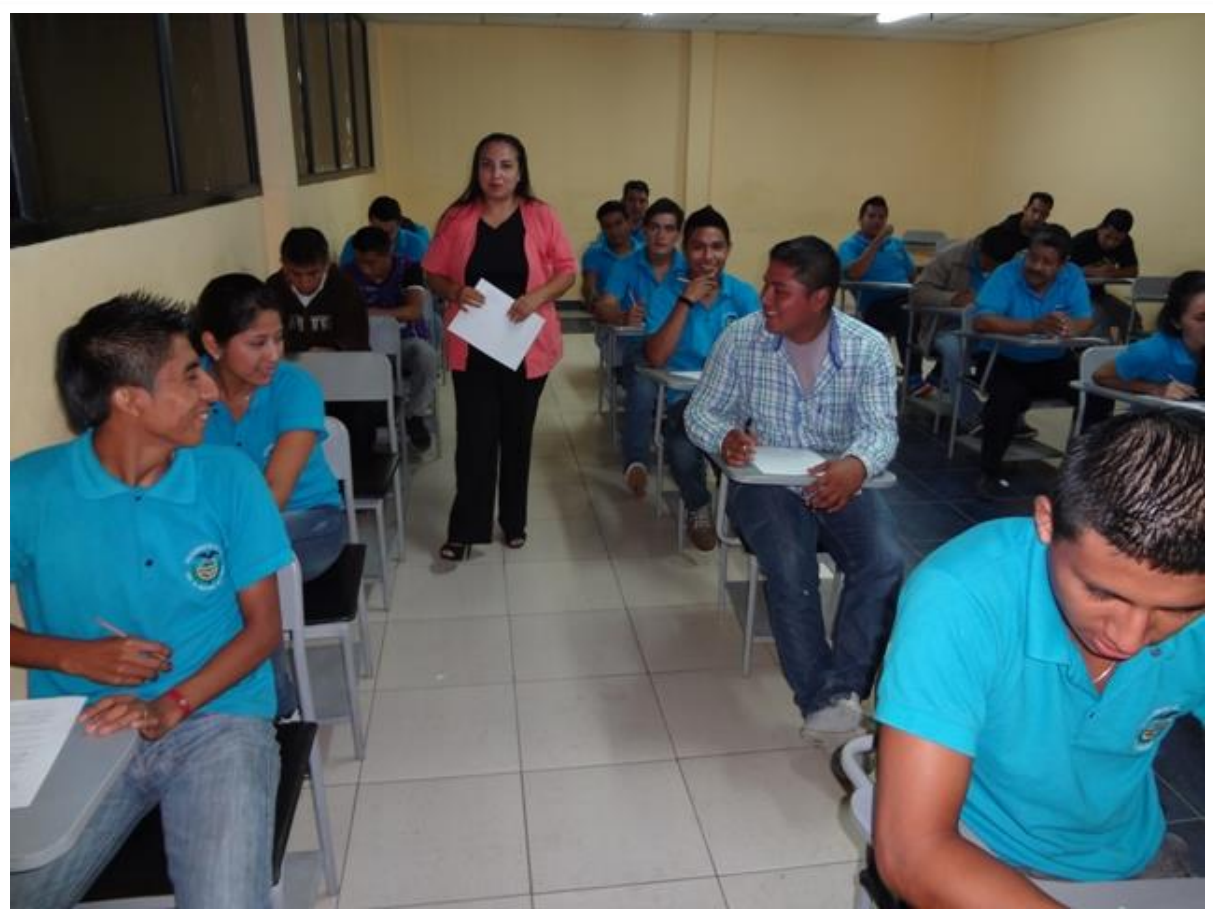

Autor: Sindicato de Choferes Profesionales del Cantón La Maná

Fuente de imagen: http://www.sindicatodechoferes/amana.com/images/galeria/DSC01663.JPG

La idea de que estas autoescuelas posean un sistema informático para la gestión educativa y legal, es obtener resultados, así como en las autoescuelas extranjeras ya mencionadas, lo cual 
sería beneficioso tanto para los que conforman estas autoescuelas como para los moradores del cantón. Con este sistema podría ser posible entregar conductores y choferes con una mejorada habilidad de conducción, que tengan los conocimientos necesarios para transitar y llevar una armonía con los peatones y ciclistas.

\section{Sistema de Gestión.}

Se mostrará a continuación como va a ir el sistema de gestión informático que llevaran las autoescuelas para su desempeño tanto en los alumnos como en los profesores e instructores. Mediante una red conceptual el cual cumplirá con los parámetros, y así también cumpliendo el propósito principal de esta idea.

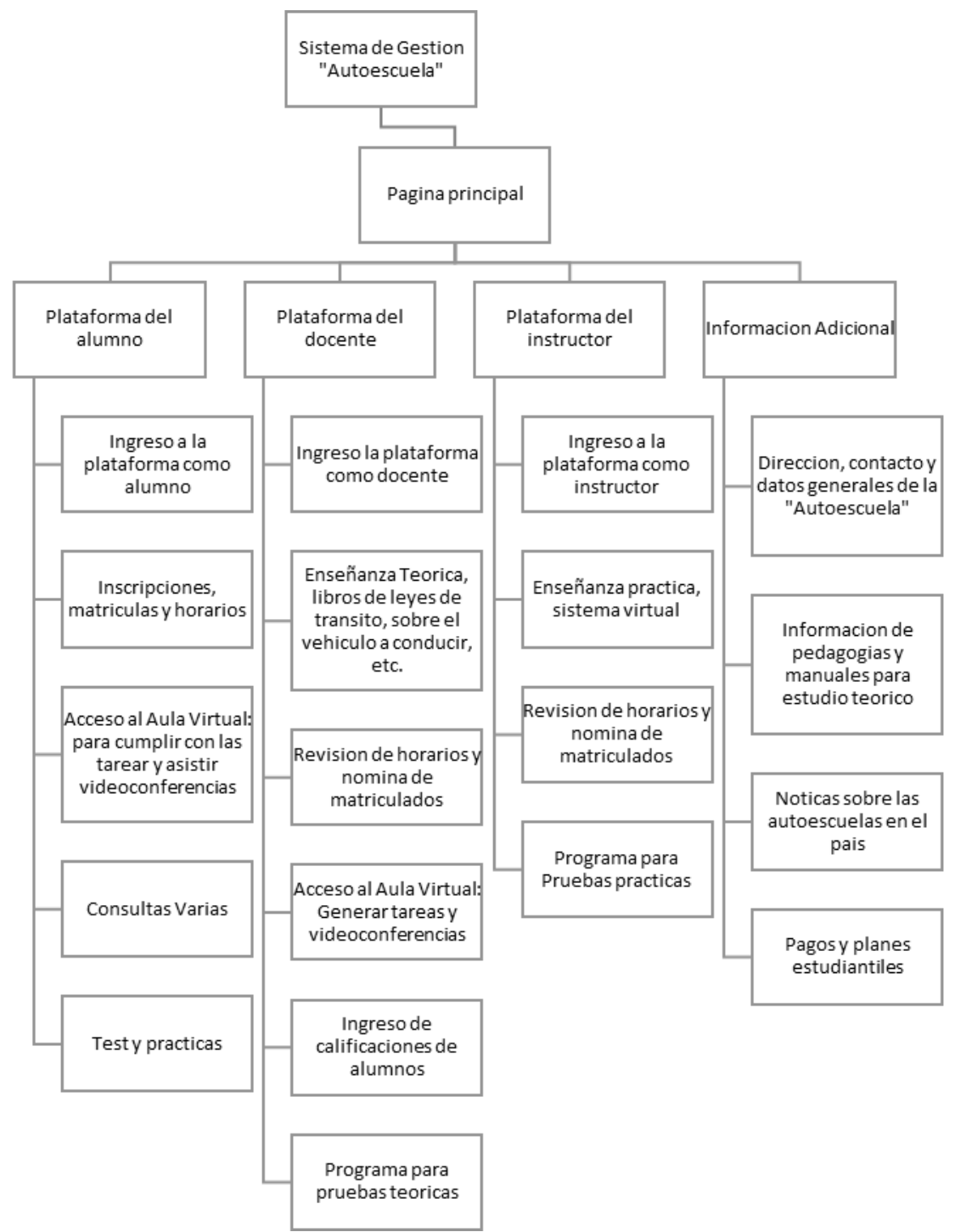

Figura 2. Esquema del sistema de gestión informático "Autoescuela", esquema realizado por el autor de este ensaño, en el cual muestra, la idea de cómo va a ser este sistema de gestión para las escuelas de conducir. Este esquema y des conceptuales y mejora la idea.

\section{Conclusiones.}


$\overline{\mathrm{Al}}$ haber realizado los estudios pertinentes para solventar la factibilidad y viabilidad de este proyecto de investigación, se ha llegado a las siguientes conclusiones.

- Un sistema informático, es un tema a nivel mundial que es muy poco o mal utilizado. Aun conociendo que este puede beneficiar a cualquier índole manipulando la información que vaya a llevar esta, realizando un tratamiento, análisis y control del mismo que, en algún posible caso, la información física presente ciertos agentes que afecte su contenido.

- La autoescuela es un factor inicial para cualquier alumno deseoso de aprender a conducir cualquier vehículo automotor, dándole el beneficio de una educación tanto teórica como practica para su educación vial, siempre tomando en cuenta que se estará llevando un control del mismo, con la finalidad de otorgarle su certificado ya sea para uso personal o en el ámbito profesional.

- Se puede destacar que la gestión mediante un sistema informático es necesario para una autoescuela, por los diferentes problemas que fueron conllevando dentro de la misma, este sistema mostrara cambios a que muchas personas y a los que conforman estas instituciones, dando el resultado de reducción, no total, de los accidentes de tránsito.

\section{Referencias.}

Anónimo. (Mayo de 2013). Blog de la Autoescuela Edición América. Obtenido de http://www.blogdelaautoescuela.com/america/tag/ecuador/

Anónimo. (18 de octubre de 2018). Ecured, Conocimiento con Todo y para Todos. Obtenido de Ciencias Aplicadas y Tecnologías, Informática: https://www.ecured.cu/Sistema

Anónimo. (19 de octubre de 2018). Ecured, Conocimiento con Todos y para Todos. Obtenido de Ciencias Aplicadas y Tecnologías, Informática: https://www.ecured.cu/Inform\%C3\%A1tica

Anónimo. (20 de Octubre de 2018). Ecured, Conocimiento con Todos y para Todos. Obtenido de Informática: https://www.ecured.cu/Sistema_inform\%C3\%A1tico

Anónimo. (2018). Instituto de Andaluz de Administración Publica. Obtenido de Junta de Andalucía:

http://www.juntadeandalucia.es/institutodeadministracionpublica/publico/anexos/e mpleo/c2.1000/TEMA\%2011.pdf

Aransay Azofra, J. M. (2018). Iberus. Obtenido de Universidad de la Rioja: https://www.unirioja.es/cu/jearansa/1011/ficheros/Tema_1.pdf

Bosque Pérez, G., \& Fernández Rodríguez, P. (2016). Principios de Diseño de Sistemas Digitales. Lejona, España: Servicio Editorial / Argitalpen Zerbitzua UPV/EHU.

Castillo Blasco, L. (20 de Enero de 2005). Universidad de Valencia. Obtenido de Universitat de València Web Site: https://www.uv.es/macas/9.pdf

Chacón, J. F. (12 de Enero de 2017). Preparadores de Oposiciones. Obtenido de PreparadoresdeOposiciones.eu: https://www.preparadores.eu/temamuestra/PTecnicos/PComerciales.pdf 
Doña Ibáñez, D. (2018). Departamento de Informática. Obtenido de Danieldona.com: https://www.danieldona.com/informatica\%20basica/2\%20sistemas\%20informatico s.pdf

Flores Corzo, V. (13 de Junio de 2018). Circula Seguro. Obtenido de CirculaSeguro.com: http://www.circulaseguro.com/aprendera-conducir-para-aprobar-el-examenpractico/

HellHacker. (7 de Septiembre de 2017). Universidad Nacional de La Plata. Obtenido de http://www.graduada.unlp.edu.ar/blog/wp-content/uploads/2017/09/completomanual-de-informtica.pdf

Kuri Abdala, J. A. (2018). Fundación Plone. Obtenido de Departamento de Ingeniería de Sistemas, Planeación y $\quad$ Transporte: http://dicyg.fic.unam.mx:8080/sistemas/publicaciones/TEMAII.5.pdf

Moriano Expósito, A. M. (2 de Noviembre de 2016). Autoescuela FOCOSUR. Obtenido de autoescuelafocosur.es:

http://autoescuelafocosur.es/images/pdf/CAP1CALIDAD.pdf

Villazán Olivarez, F. J. (1 de Noviembre de 2010). Universidad Pedro de Gante. Obtenido de https://www.upg.mx/wp-content/uploads/2015/10/LIBRO-31-Manual-deInformatica.pdf

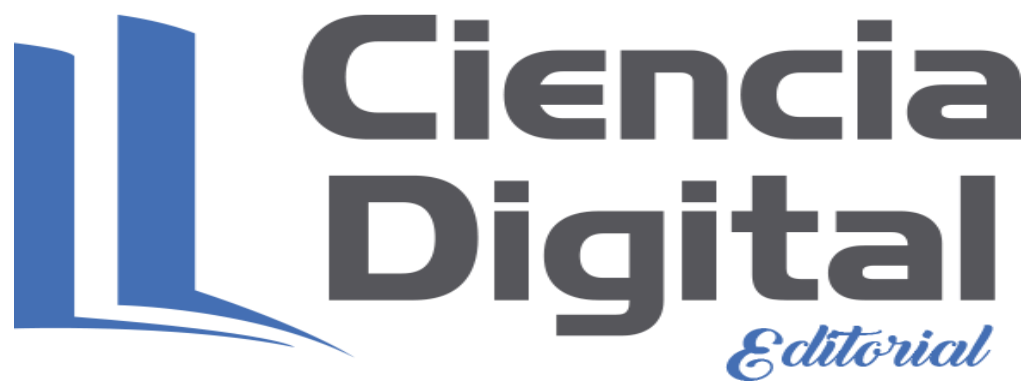




\section{Para citar el artículo indexado.}

Velasteguí E. \& Bravo B. (2017). Por medio de un sistema informático en las escuelas de conducción. Revista electrónica Visionario Digital 1(1), 21-33. Recuperado desde: http://cienciadigital.org/revistacienciadigital2/index.php/VisionarioDigital/article/view/256 $\underline{1558}$

\section{【Ciencia}

El artículo que se publica es de exclusiva responsabilidad de los autores y no necesariamente reflejan el pensamiento de la Revista Ciencia Digital.

El articulo queda en propiedad de la revista y, por tanto, su publicación parcial y/o total en otro medio tiene que ser autorizado por el director de la Revista Ciencia Digital. 